\title{
MRI Head Coil Malfunction Producing Artifacts Mimicking Malformation of Cortical Development in Pediatric Epilepsy Work-Up
}

\author{
(D) N. Kashani, (D) N. Khan, (D).M. Ospel, and (D) X.-C. Wei
}

\section{ABSTRACT}

SUMMARY: We recently observed a type of MR imaging artifact that consistently mimics an abnormal appearance of the cerebral cortex, leading to initial misinterpretation and repeat scans. The artifact is caused by malfunction of part of the multichannel phased array head coil and is manifested by irregularity of cortical surface and gray-white matter junctions. The presence of such an artifact can be confirmed by assessing the background noise of the MR images and checking the coil element status on the MR imaging operator console.

$E^{2}$ valuation of the morphology of the cerebral cortex in pediatric neuroimaging is crucial to rule out a malformation of cortical development. The cortical abnormalities can be subtle, and utilization of multiple-phased array head coils is beneficial. ${ }^{1}$ Modern MR imaging scanners often use phased array coils that consist of multiple detectors, which allow images of high quality. ${ }^{2,3}$ These coil elements are placed around the body parts being imaged (Fig $1 A)$. They are usually divided into groups that are, in turn, connected to the system by plugging into the scanner. ${ }^{3}$ The operators can view the locations and status of each group of coil elements on the computer monitors (Fig 1B).

Recently, we encountered a series of incidents in which the 2 anterior groups of coil elements were inadvertently disconnected from the MR imaging system during patient scanning. This malfunction could not be eliminated by the scanner operator, neither could it be identified in the DICOM header. The resulting artifact occurred exclusively anteriorly and bilaterally and can be mistaken for a cortical abnormality. In the first incident, the scan was interpreted by neuroradiologists as possible polymicrogyria until a repeat scan 3 weeks later showed a normal cerebral cortex, whereas in the following incidents, the neuroradiologists recognized the artifact, thereby avoiding misdiagnosis.

Received March 15, 2020; accepted after revision May 12

From the Department of Radiology (N. Kashani. N. Khan, J.M.O.), University of Calgary, Calgary, Alberta, Canada; and Diagnostic Imaging (X.-C.W.), Alberta Children's Hospital, Calgary, Alberta, Canada.

Please address correspondence to Xing-Chang Wei, MB, MSc, FRCPC, 2888 Shaganappi Trail NW, Calgary, AB T3B 6A8, Canada; e-mail: weix@ucalgary.ca; @johanna_ospel

Indicates article with supplemental on-line photos.

http://dx.doi.org/10.3174/ajnr.A6639

\section{Case Presentation}

Case 1. A 2-week-old term male neonate was admitted with suspected bacterial meningitis. A brain MR imaging was requested to rule out any complications from meningitis. The MR imaging was performed (3T Magnetom Vida; Siemens), including 3D MPRAGE T1WI, axial and coronal FSE T2WI, DWI, gradient-echo T2*weighted images, TOF-MRA, and phase-contrast MRV. A 20-channel head coil was used. A small amount of pus in the trigones of both lateral ventricles was identified, and bilateral frontal cerebral cortex surfaces as well as cortical white matter junctions appeared irregular, while their signal intensities were normal on all sequences. The findings were interpreted as possible polymicrogyria (Fig $2 A,-C$ ).

Repeat MR imaging was performed 20 days later on the same scanner with identical sequences and coils. The frontal cerebral cortex now appeared normal, and the previously seen irregular outer and inner surfaces of the frontal cortex were no longer present (Fig $2 E,-G)$. Therefore, a coil malfunction was suspected. Scanning parameters of the first scan were then reviewed, and it was noted that the anterior 2 groups of the coil elements were "off."

Case 2. In a 4-year-old boy with developmental delay, chromosomal microdeletion, suspected clinical seizures, and abnormal electroencephalogram findings, a brain MR imaging was performed (3D MPRAGE sequence on the same clinical scanner as in case 1). The MR imaging technologist noted the irregular surfaces of the frontal lobes (On-line Fig $1 A$ ) and increased background noise in the coil elements that were at "off" status. A repeat 3D MPRAGE was performed after re-plugging the head coil, and the bifrontal cortical irregularity and background noise were no longer seen (On-line Fig $1 C,-D)$.

Case 3. In an 8-year-old boy with familial Li-Fraumeni syndrome, MR imaging without contrast was performed to exclude intracranial neoplasms (3T MPRAGE T1-weighted images obtained from 


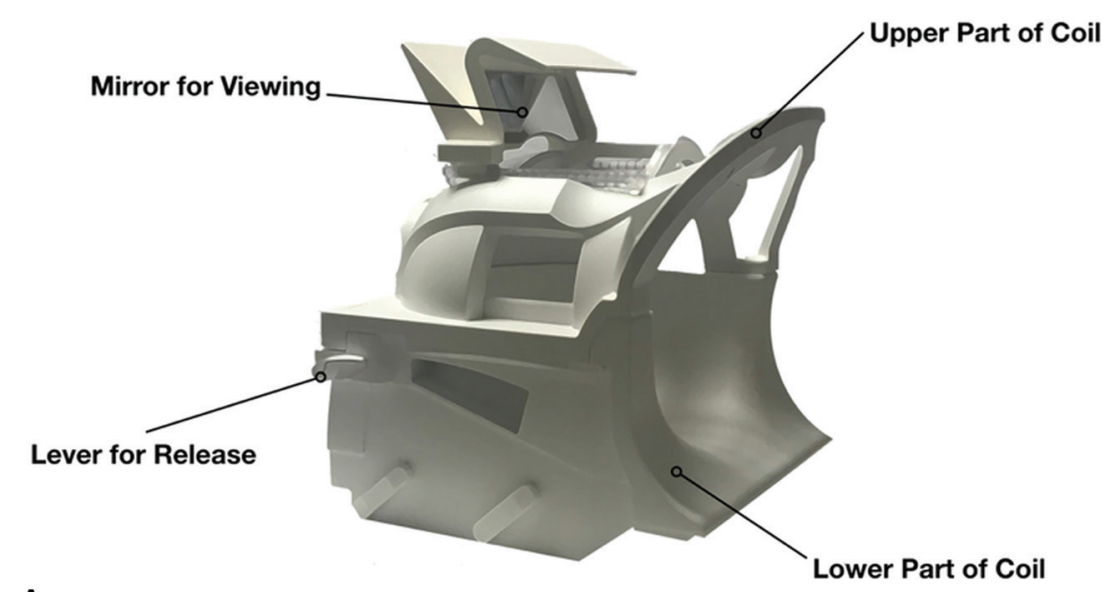

A

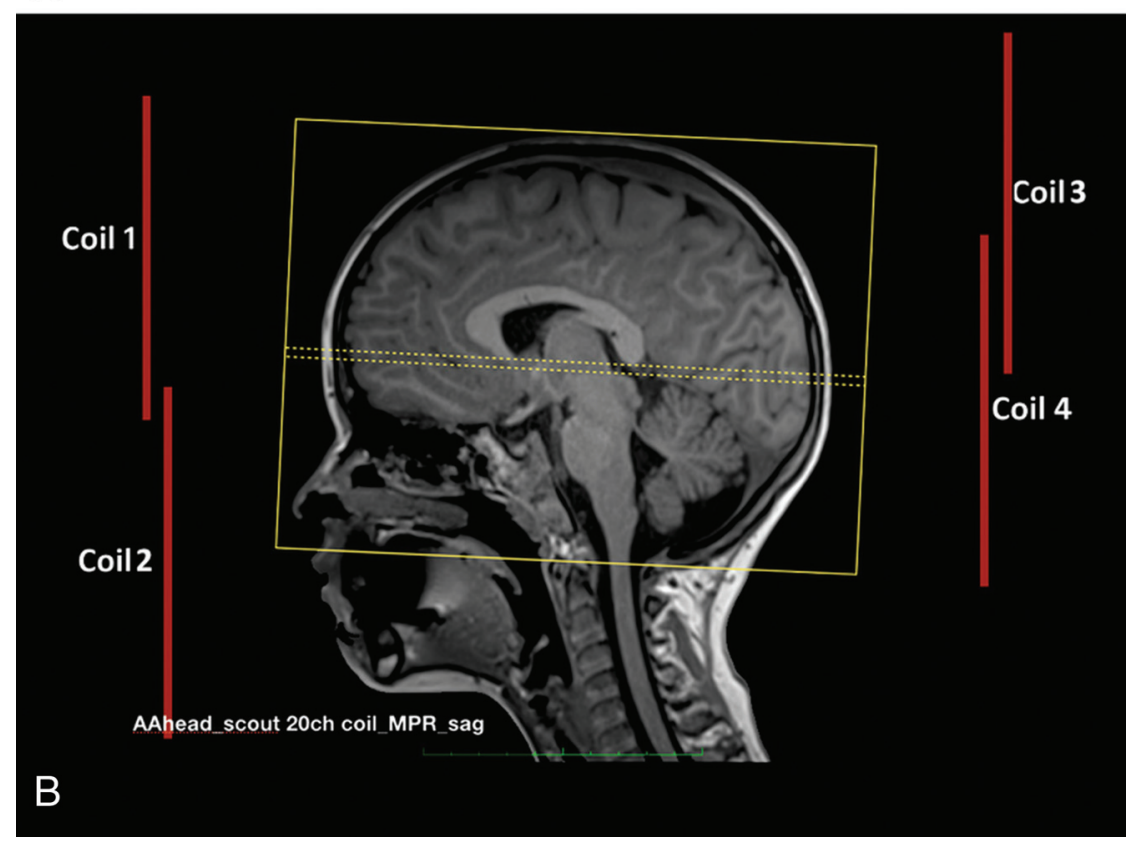

FIG 1. Picture of a typical multichannel MR head coil (A) shows the positions of the multiple coil elements. A modified screenshot from the operator console of the system $(B)$ shows indicators of the positions of the 4 groups of a 20-channel head coil. Red bars show the position of the individual groups of head coil elements. The coils 1 and 2 are anterior coils, while the coils 3 and 4 are posterior coils.

the same scanner with the same head coil as in cases 1 and 2); irregular surfaces and gray-white matter junctions were noted on these scans (On-line Fig 2A). An MR imaging with contrast was performed 3 days later on another scanner (3T Discovery MR750w; GE Healthcare) with a 32-channel head coil, and no cortical irregularity was seen (On-line Fig 2C). The status of the head coil was checked, and in the first scan, the frontal 2 groups of the coil elements were at "off" status.

\section{DISCUSSION}

Artifacts on MR imaging can mimic pathology and lead to confusion, misdiagnosis, and unnecessary investigations. While some MR imaging artifacts are caused by patient factors such as motion of the body and/or internal structures during a prolonged scan, others can be attributed to the hardware devices used in image acquisition. Recently in our practice, we observed a peculiar type of MR imaging artifact on a 3T scanner that causes apparent irregularity of the cerebral cortex, mimicking an abnormal appearance of the cerebral cortex and leading to initial misinterpretation. This type of artifact was caused by a loosened connection of the anterior 2 groups of the multichannel head coil. The authors could not find any description of such an artifact in the English language literature.

When multichannel head coils are used, the proximity of the coil elements to the surface of the patient (and ROI) is critical because the radiofrequency coil depends on the near field to couple energy from the spin system to the coil elements. When the radiofrequency coil is away from the ROI, reduced coupling to the spin system and poor signal-to-noise ratio occur. ${ }^{4}$ When the anterior coil elements malfunction, the frontal brain structures are relatively remote from the functioning posterior receiver coil elements. Therefore, the anterior portion of the MR images will have a much lower signal-to-noise ratio, and the frontal lobes of 

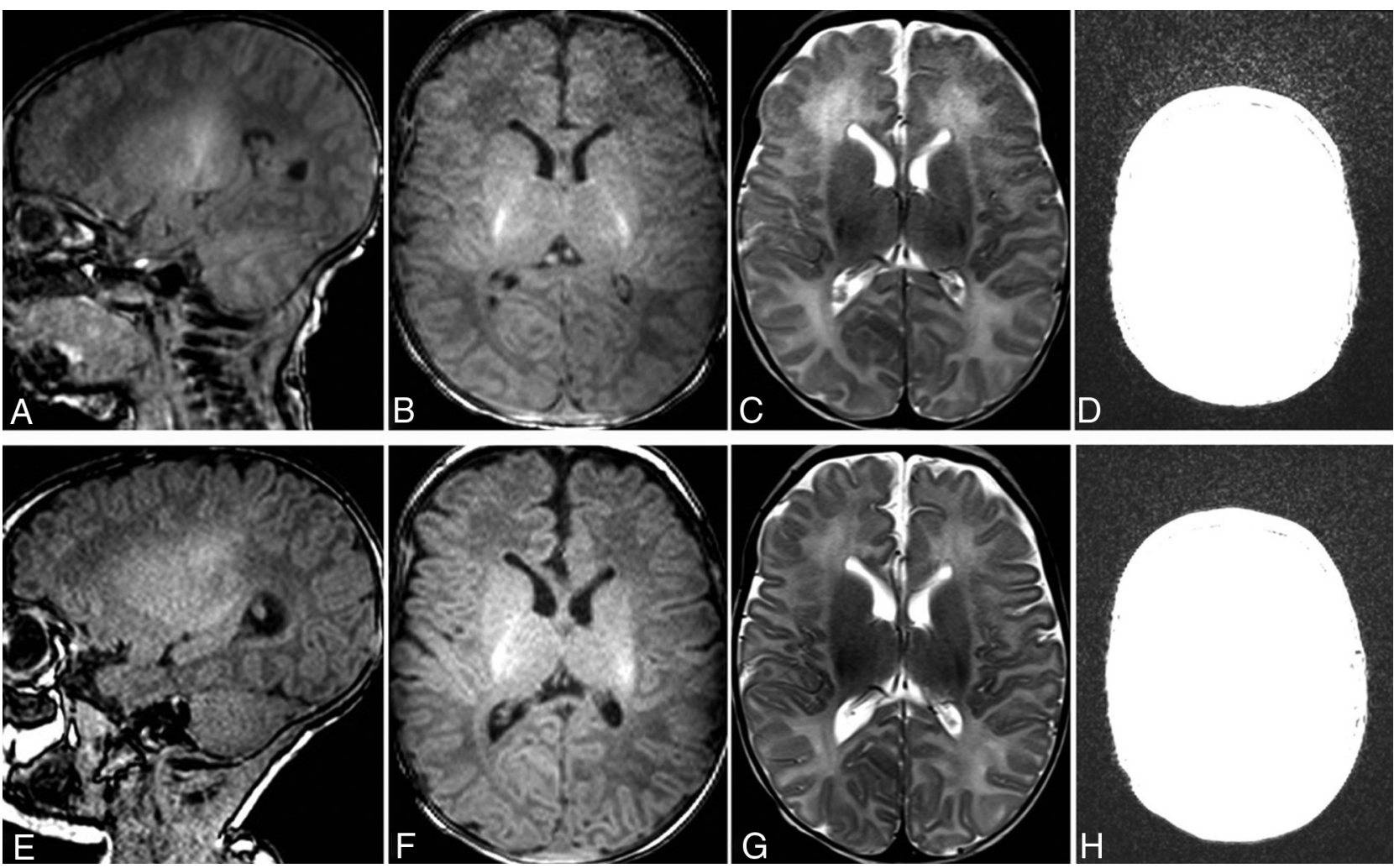

FIG 2. MR imaging of the brain of case 1 at presentation $(A-D)$ and 20 days later $(E-H)$. On the sagittal and axial TTWI $(A$ and $B)$ and the axial T2WI (C) of the first scan, the cerebral cortices of the bilateral frontal lobes show apparent irregular surfaces and gray-white matter junctions. Because the irregularity was observed on both TTWI and T2WI and the posterior cerebral cortices have rather smooth surfaces, without knowing the possibility of coil malfunction, we interpreted the findings as possible polymicrogyria. On the sagittal and axial TWI ( $E$ and $F)$ and the axial T2WI (G) of the follow-up scan obtained only 20 days later, the irregularity is no longer present, suggesting that the irregular appearance of the cortex seen on the first scan is artifactual rather than pathologic. When the window setting was adjusted to evaluate the background noise of the axial TWWI of the first $(D)$ and follow-up $(H)$ scan, it is obvious that the first scan has pronounced background noise in the frontal part of the image.

the brain will show apparent irregular surfaces and an irregular gray-white matter junction. While the malfunction of a coil may produce substantial artifacts on $1.5 \mathrm{~T}$ MR imaging scanners, the artifacts may be considerably more subtle on 3T MR imaging scanners. On high-field MR imaging, because of the overall high signal-to-noise ratio, the drop of the SNR may not be severe enough to alarm the MR imaging technologist about possible hardware malfunction.

The apparent cortical irregularity simulates malformation of cortical development. Indeed, we misinterpreted this artifact as a polymicrogyria in our first patient. Fortunately, the patient had a repeat MR imaging not long after, and no harm was done to the patient. MR imaging technologists and radiologists should be vigilant for this type of artifact. Unlike other MR imaging artifacts, this type of artifact persists and appears on all the sequences of any acquisition plane if not recognized. The radiologist should not conclude that the apparent cortical irregularity is not artifactual because the cortical irregularity can be seen on all sequences. Bilateral and symmetric abnormalities should raise suspicion for the presence of such an artifact. It can be easily confirmed by adjusting the window setting of the images and visualization of a pronounced frontal background noise (Fig $2 D$ and On-line Figs $1 B$ and $2 B$ ). The artifact can be corrected by re-plugging the head coil. Of note, our case series consists of only 3 patients, and the artifact we described should ideally be confirmed in a prospective fashion in further patients.

\section{ACKNOWLEDGMENTS}

The authors thank Martin Sherriff and Anvita Pauranik for their help and support.

Disclosures: Johanna Maria Ospel-UNRELATED: Grants/Grants Pending: University of Basel Research Foundation, Julia Bangerter Rhyner Foundation, and Freiwillige Akademische Gesellschaft Basel, Comments: research scholarships.

\section{REFERENCES}

1. Raybaud C, Widjaja E. Development and dysgenesis of the cerebral cortex: malformations of cortical development. Neuroimaging Clin N Am 2011;21:483-543 CrossRef Medline

2. Parikh PT, Sandhu GS, Blackham KA, et al. Evaluation of image quality of a 32-channel versus a 12-channel head coil at $1.5 \mathrm{~T}$ for MR imaging of the brain. AJNR Am J Neuroradiol 2011;32:365-73 CrossRef Medline

3. Seiberlich N, Jordan DW. Imaging principles in magnetic resonance imaging. In: Boll DT, Haaga JR, eds. CT and MRI of the Whole Body. 6th ed. Elsevier; 2017:46-68

4. Duensing R, Fitzsimmons J. 3.0 T versus 1.5 T: coil design similarities and differences. Neuroimaging Clin N Am 2006;16:249-57 CrossRef Medline 